\title{
A Novel Protocol for Transparent and Simultaneous Spectrum Access between the Secondary User and the Primary User in Cognitive Radio Networks
}

\author{
Jonathan Backens ${ }^{\mathrm{a}, *}$, Chunsheng Xin ${ }^{\mathrm{b}}$, Min Song ${ }^{\mathrm{c}}$ \\ ${ }^{a}$ Christopher Newport University, Department of Physics, Computer Science and \\ Engineering, Newport News, VA 23606, USA. \\ ${ }^{b}$ Old Dominion University, Department of Electrical and Computer Engineering, Norfolk, \\ VA 23435, USA. \\ ${ }^{c}$ Michigan Technological University, Department of Computer Science, Houghton, MI \\ 49931, USA
}

\begin{abstract}
In this paper, we propose a novel protocol for cognitive radio networks, termed spectrum co-access protocol (SCAP), for secondary users to transparently and simultaneously access spectrum with primary users. The motivation is to eliminate the disruption to secondary user communications by the resurgence of primary user transmission. This protocol enables mutually beneficial coexistence between the primary user network and the secondary user network. Through spectrum co-access, SCAP creates a virtual secondary user control channel in licensed spectrum that is invisible to the primary user. As a result, SCAP is a medium access control protocol that allows for simultaneous spectrum access between the secondary user and the primary user networks. The performance evaluation indicates that SCAP provides significant performance improvement for the secondary user network over the existing opportunistic spectrum access scheme.
\end{abstract}

Keywords: cognitive radio networks, spectrum co-access, simultaneous spectrum access.

\footnotetext{
A preliminary version of this work was presented in ICCCN 2014 [1].

* Corresponding author

Email addresses: jonathan.backens@cnu.edu (Jonathan Backens), cxin@odu.edu (Chunsheng Xin), mins@mtu.edu (Min Song)

Preprint submitted to Elsevier Computer Communications (ComCom)

June 16, 2015
}

(C) 2015. This manuscript version is made available under the Elsevier user license http://www.elsevier.com/open-access/userlicense/1.0/ 


\section{Introduction}

Providing access to licensed spectrum for secondary user (SU) networks has become a major area of research in the last decade. This has been motivated by the well documented under-utilization of licensed spectrum and the growing interest in exploiting "white spaces" or unused spectrum. Research on the SU spectrum access can be categorized either as geo-location based spectrum access $[2,3,4,5,6,7]$, or opportunistic spectrum access $[8,9,10,11,12,13,12,14]$. Geo-location based spectrum access relies on primary users (PUs) exhibiting spatially and/or temporally predictable behavior. If PU behavior does not exhibit predictability, then spectrum sharing often falls into opportunistic spectrum access, where secondary users (SUs) perform spectrum sensing to dynamically detect PU channel access, and opportunistically access the channel during the period between two PU transmissions.

Recently there has been significant interest on medium access control (MAC) protocols for CRNs. As every new method for accessing licensed spectrum is proposed, soon the counterpart MAC protocols are developed. A discussion of some the current challenges and the most popular cognitive radio MACs can be found in [15]. One of the major challenges to MAC protocols for SUs has been the inability of SU networks to remain active during PU transmissions. That is, SUs can be active on a licensed channel only during periods of PU inactivity. Thus, the arbitrary resurgence of the PU signal on a licensed channel interrupted the ongoing $\mathrm{SU}$ communications, and can significantly degrade the performance SU networks.

An SU spectrum access protocol that enables SU transmission simultaneous to PU transmissions would clearly be beneficial. Generally such a protocol would require coordination between PU and SU networks, e.g., changes to the existing PU access scheme and/or codebook, which is usually not desirable by the PUs. In this paper, we aim to design a transparent spectrum access protocol such that SUs can access licensed spectrum without requiring any change to the existing PU access protocol or codebook and do not degrade the PU performance. We call such SU spectrum access as transparent and simultaneous to the PU. Note that here the PU can be aware of SU transmissions, but the latter does not 
have negative effect on the PU transmission. In this paper, we will show that certain types of PU networks can support such transparent and simultaneous $\mathrm{SU}$ transmissions. These transmissions were proven to be possible under certain scenarios and represent a significant step in realizing spectrum co-access between PU nodes and SU nodes. However, not all SU nodes can meet the requirements for spectrum co-access. These unqualified SU nodes will once again only be able to transmit in the absence of PU activity. Thus a MAC protocol for SUs is needed to facilitate both transparent spectrum co-access during PU activity, and spectrum access for all SU nodes during PU absence.

With a large number of SUs attempting to access a limited number of PU transmission gaps, there is another problem of scheduling $\mathrm{SU}$ transmissions. Typically SU networks rely on two basic techniques for solving this problem. First they may use a designated control channel that is not affected by the PU to schedule transmissions among SU nodes $[16,17,18]$. Second, if a designated control channel is not available, SUs typically utilize some channel hopping algorithm to guarantee a reliable SU control channel [9, 19, 20, 21]. However all those protocols have been designed for the opportunistic spectrum access and cannot be used for spectrum co-access for SUs.

In this paper, we present a spectrum co-access protocol (SCAP) that addresses both the concerns of opportunistic spectrum access and the challenge of facilitating fair SU channel sharing. First we will show that in the case of multihop PU networks that use relaying, a subset of SUs will be able to co-access the licensed spectrum simultaneous with PU transmissions. This means that the PUs will be either unaware of the SU transmissions, i.e., without performance degradation, or they will benefit from spectrum co-access with an increase in SINR which results in a higher achievable rate. Hence we can consider these SU transmissions through spectrum co-access as a virtual communication channel. It can be used for both SU message transport and an SU broadcast control channel.

The remainder of the paper is organized as follows. Section 2 provides an overview of a dynamic spectrum access architecture for which the SCAP protocol was designed. Section 3 classifies SUs based on their capability for spectrum co-access. Section 4 describes the proposed SCAP protocol in details. The 
performance evaluation is presented in Section 5 . Section 6 concludes the paper.

\section{Overview of the DSCA Architecture}

Although conceived as very difficult for a long time, co-accessing licensed spectrum by SUs together with PUs is still possible. In [22], we have proposed a Dynamic Spectrum Co-Access (DSCA) architecture for cognitive radio networks. The SCAP protocol is designed for the DSCA architecture. For the completeness of this work, we give a brief introduction of the DSCA architecture in this section.

Fig. 1 illustrates the simultaneous spectrum access between a pair of PU transmitter and receiver, and a pair of SU transmitter and receiver in a normalized Gaussian path loss $(1, a, b, 1)$ channel, where $a$ and $b$ denote the normalized path losses from the SU transmitter to the PU receiver, and from the PU transmitter to the SU receiver, respectively. The PU transmitter sends a packet to the PU receiver. Assume that the SU knows the PU packet a priori. While this assumption of the SU transmitter having a priori knowledge of PU packet is a challenge, DSCA smartly utilizes overhearing during the PU packet forwarding in PU multi-hop networks to obtain such information. For example, as illustrated in Fig. 3 in the next section, when PU2 receives a PU packet from its upstream node PU1, SU1 also overhears the PU packet from PU1. Then when PU2 relays the packet to PU3, SU1 has a priori knowledge of this packet, and can co-access spectrum with PU2. A main idea of the DSCA architecture is to let the SU transmitter use a portion of its power to transmit the PU packet to boost the SINR at the PU receiver, so that PU is incentivized for simultaneous spectrum access with the SU.

Let $P_{p}$ and $P_{s}$ denote the transmit power of the PU and SU transmitters, respectively. Let $\gamma$ denote the portion of the power of the $\mathrm{SU}$ transmitter to transmit the PU packet and $(1-\gamma)$ the portion of power to transmit its own packet, as illustrated in Fig. 1. It can be shown that the PU received signal power (excluding interference) is $\left(\sqrt{P_{p}}+a \sqrt{\gamma P_{s}}\right)^{2}$, which is larger than the PU received signal power $P_{p}$ without spectrum co-access of SU. On the other hand, it can be shown that the total noise at the PU receiver (including channel noise 


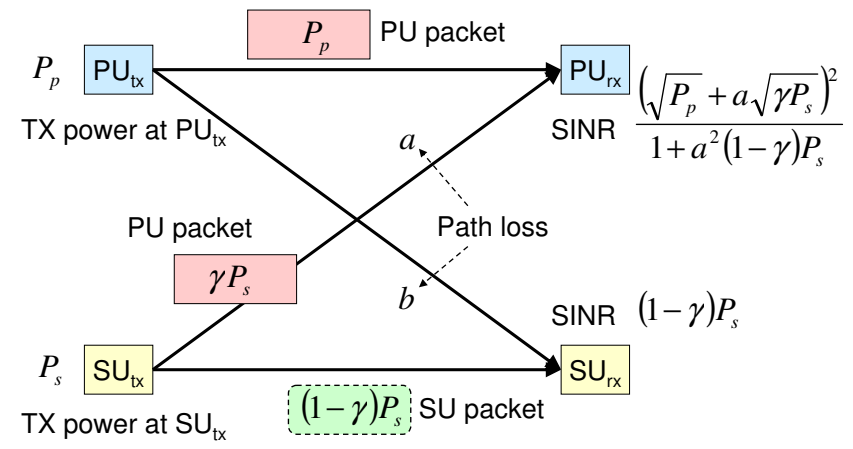

Figure 1: Simultaneous spectrum access between a PU link and an SU link

and $\mathrm{SU}$ transmission) is $1+a^{2}(1-\gamma) P_{s}$. The SINR at the PU receiver with spectrum co-access by the $\mathrm{SU}$ is thus

$$
\frac{\left(\sqrt{P_{p}}+a \sqrt{\gamma P_{s}}\right)^{2}}{1+a^{2}(1-\gamma) P_{s}}
$$

One may note that the noise at the PU receiver is higher than the case without spectrum co-access, due to the SU transmission of the SU code word. However, as long as the SINR is larger than $P_{p}$, i.e.,

$$
\frac{\left(\sqrt{P_{p}}+a \sqrt{\gamma P_{s}}\right)^{2}}{1+a^{2}(1-\gamma) P_{s}} \geq P_{p},
$$

where $P_{p}$ is the SINR without spectrum co-access (with normalized path loss 1 ), the achievable rate for the PU is not degraded by the spectrum access. By adjusting $\gamma$, we can actually increase the achievable rate for the PU and hence provide incentives to the PU for spectrum co-access.

On the other hand, utilizing the dirty paper coding technique [23], the interference from the PU transmission to the SU receiver can be canceled, and hence the normalized channel noise is 1 at the $\mathrm{SU}$ receiver. It can be shown that the received signal power at the SU receiver is $(1-\gamma) P_{s}$. Thus the SINR at the SU receiver is $(1-\gamma) P_{s}$. Hence by setting $\gamma$ to an appropriate value, the DSCA architecture also guarantees a minimum achievable rate for the $\mathrm{SU}$ to participate in spectrum co-access (to help the PU to boost SINR). Therefore, the spectrum co-access in the DSCA architecture is not only possible, but mutually beneficial 
to both the PU and the SU.

However, there is a trade-off for setting $\gamma$. To offer more incentives to the PU, a larger $\gamma$ value is needed. On the other hand, to maximize the $\mathrm{SU}$ performance, a smaller $\gamma$ is desirable. In the DSCA architecture, a parameter $K$ for the $\mathrm{PU}$, termed co-access incentive or co-access constraint, is defined to be the percentage SINR increment at the PU receiver that the SU must provide to be allowed for simultaneous spectrum access. That is, the PU co-access constraint $K$ satisfies the following equation.

$$
\frac{\left(\sqrt{P_{p}}+a \sqrt{\gamma P_{s}}\right)^{2}}{1+a^{2}(1-\gamma) P_{s}} \geq P_{p}(1+K) .
$$

From (1), the minimum $\gamma$ to offer the PU co-access constraint $K$ is given as

$$
\gamma \geq\left(\frac{\sqrt{\left(P_{s}\left(K+P_{p}\right)\left(K+a^{2} P_{s}\left(1+P_{p}+K\right)+1\right)\right)}-\sqrt{P_{p} P_{s}}}{a P_{s}\left(K+P_{p}+1\right)}\right)^{2} .
$$

Note that although the PU requires an increase $K$ in SINR to allow the SU to co-access spectrum, it is done transparently to the PU and thus does not require any change in the PU side. Similarly, the SU co-access constraint $\lambda$ is

defined as the minimum received SINR at the SU receiver that is desired by the $\mathrm{SU}$ for participating in spectrum co-access, i.e., we must have $(1-\gamma) P_{s} \geq \lambda$. Thus we have the upper range of $\gamma$ as follows.

$$
\gamma \leq 1-\frac{\lambda}{P_{s}} .
$$

At last, given parameters $K$ and $\lambda$, and the path loss, we can find the value range of $\gamma$ for a pair of SU transmitter and receiver to co-access spectrum with a pair of PU transmitter and receiver, based on (2) and (3).

\section{Secondary User Co-Access Capability Classification}

As discussed in the preceding section, under certain constraints $(K, \lambda$, and path loss) an SU link is able to co-access a licensed channel simultaneously with a PU link. These constraints help to determine how much of the power the 
transmitter of a given SU link should use to boost the PU transmission. The constraints are relevant to the location of the SU transmitter in relation to the PU transmitter and receiver. Furthermore, a particular SU link qualifying for co-access is not automatically symmetric. That is, it is possible that the SU transmitter of an SU link can co-access spectrum while the SU receiver of the link is not able to. However, in wireless communications, acknowledgment (ACK) packets are usually needed to verify that transmitted packets are received, as the bit errors are more likely than in wired communications. Thus in order for a pair of SU nodes to successfully co-access with a PU link, constraints must be placed on both the SU transmitter to send the data packet, and the SU receiver to send the ACK packet back to the transmitter. There are two possible scenarios for successful SU receiver ACK. First of all, if the SU receiver meets the same co-access constraints as the SU transmitter, then it can send the ACK packet during the PU transmission. The second possibility is if the $\mathrm{SU}$ receiver can meet the constraints necessary to co-access spectrum together with the PU receiver ACK transmission, then it could transmit the SU ACK packet during the PU ACK transmission.

We classify the SU nodes into three types based on whether they are able to co-access spectrum with the PU to transmit data packets, or ACK packets. The first type of SU nodes are the transmitters of SU links that are fully co-accessible with a PU link, and are termed co-access nodes. This means that they meet all of the spectrum co-access constraints to send data packets. The second type of $\mathrm{SU}$ nodes are the $\mathrm{SU}$ receivers that are unable to co-access spectrum to transmit data packets, but meet the constraints to co-access spectrum to transmit ACK packets, due to their ability to predict and estimate the PU ACK packets. These SU nodes are termed co-ack nodes. The third type of SUs do not meet any co-access constraint, neither to transmit data packets nor to transmit ACK packets. This type of SU nodes will have to rely on PU transmission gaps for transmitting data or ACK packets. They are termed non-qualified nodes. We summarize the types of SU nodes as follows.

- co-access nodes: SU transmitters or receivers able to co-access spectrum with the PU nodes to transmit data (or ACK) packets. 


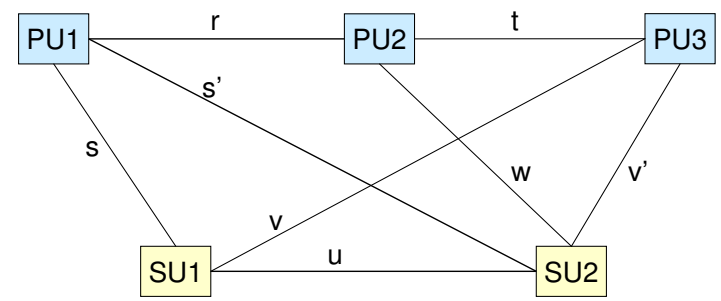

Figure 2: An SU link co-accesses spectrum with a 3-node PU network. The legend on the link is path loss.

- co-ack nodes: SU receivers able to co-access spectrum with the PU ACK packet transmissions only, to send SU ACK packets.

- non-qualified nodes: SU nodes unable to co-access the spectrum.

Next we will derive the specific conditions that must be met by the co-access and co-ack SU nodes. For the ease of description, we use Fig. 2 as an example.

The constraints for spectrum co-access to send data packets by a co-access node, e.g., SU1 in Fig. 2, are given as follows,

$$
\begin{gathered}
v \leq \frac{1-\sqrt{1-\left(K\left(\frac{t^{2} P_{P}}{N}(K+1)\left(\frac{1}{\gamma}-1\right)-1\right)\right.}}{\frac{t \sqrt{P_{p} P_{s}}}{N \sqrt{\gamma}}(K+1)(1-\gamma)-\frac{\sqrt{\gamma P_{s}}}{t \sqrt{P_{p}}}} \\
s \leq r
\end{gathered}
$$

where $v, t, s, r$, and $u$ are the path losses as shown in Fig. 2 and $K$ is the PU co-access constraint.

Next we derive the conditions that must be met by the co-ack nodes. In order for an SU receiver to be able to ACK an SU packet transmission, it too must be able to access the licensed channel simultaneously with PU transmissions. This can happen in two ways. First, the SU receiver may also be a fully qualified co-access SU and can reply to SU packet transmissions during regular PU transmission period. In such a case referring to Fig. 2 we can simply exchange $s^{\prime}$ for $s$. The other scenario is where the SU receiver cannot meet the co-access constraints, however it is able to co-access with the ACK packet transmission by the PU receiver. Specifically, if the SU receiver knows when the PU ACK pack will be transmitted, e.g., after PU TX + SIFS + DIFS in the case that 
the PU uses the IEEE 802.11 MAC protocol, it will be able to approximate the PU code word and co-access with this code word. Since the PU ACK packet for each PU data packet contains only addressing and error information, the PU ACK packets will be highly similar. The SU receiver does not need to decode the information, but only estimates the PU code word (it already knows the codebook) and then co-access spectrum with the PU ACK transmission for transmitting its own ACK. Suppose SU2 in Fig. 2 is a co-ack node. Then the constraints to estimate this PU ACK packet and co-access spectrum with it are as follows.

$$
\begin{gathered}
w \geq \frac{1-\sqrt{1-\left(K\left(\frac{t^{2} P_{p}}{N}(K+1)\left(\frac{1}{\gamma}-1\right)-1\right)\right.}}{\frac{t \sqrt{P_{p} P_{s}}}{N \sqrt{\gamma}}(K+1)(1-\gamma)-\frac{\sqrt{\gamma P_{s}}}{t \sqrt{P_{p}}}} \\
w \leq t
\end{gathered}
$$

\section{Spectrum Co-Access Protocol (SCAP)}

In this section, we describe the SCAP protocol. It is a distributed MAC protocol for the DSCA architecture to address the coordinated channel access among SU nodes in large SU networks. There are two objectives for the protocol: (1) provide fair channel access among SU nodes, and (2) maximize the number of SU nodes that access the licensed spectrum.

The SCAP protocol uses a two-phase cycle based on the activity of the PU network to schedule SU nodes to access spectrum. First, during PU access to the licensed channel, the co-access and co-ack SU nodes within the interference range of the PU transmission access the spectrum based on the constraints listed in the preceding section. These SU nodes operate under an intelligent round robin scheduling scheme termed co-access round robin (CRR). In the absence of PU transmissions, all SU nodes operate under an opportunistic access scheme relying on a modified contention window. Since the absence of the PU transmission will allow for all SU nodes (co-access, co-ack, and non-qualified SUs) to participate, this spectrum access mode is termed all $S U$ contention (ASC). In Fig. 3 an example of the SCAP operation is shown, with co-access nodes SU1, SU2 and SU3 each co-accessing with the PU relay transmissions. During the 


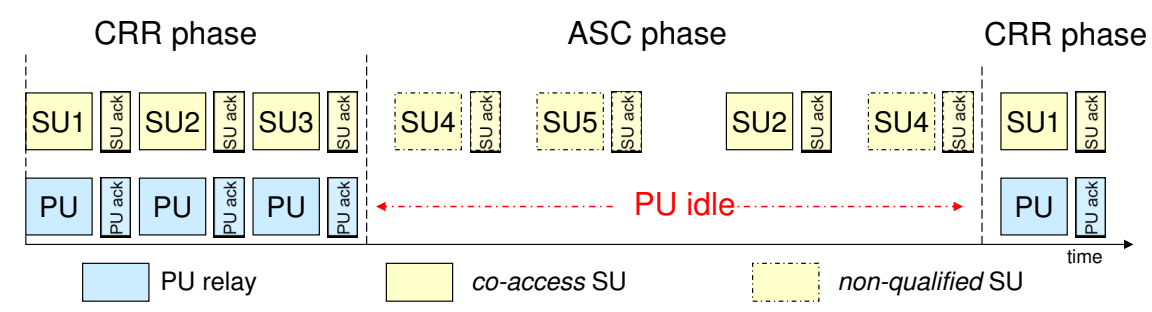

Figure 3: Spectrum co-access under the SCAP protocol, with one PU, 3 co-access SUs (SU1,SU2,SU3) and 2 non-qualified SUs (SU4, SU5)

first phase, the co-access SUs (SU1, SU2, and SU3) co-access spectrum with the PU relay using the CRR scheme. In the second phase, both the non-qualified SUs (SU4 and SU5) and the co-access SU (SU2) access spectrum using the ASC scheme, since the $\mathrm{PU}$ is inactive. The next phase is the CRR phase again. This CRR-ASC cycle repeats.

\subsection{SCAP Framework}

The framework for the SCAP protocol is shown in Fig. 4. Since the behavior of an SU node depends directly on the activity of the PU and other SUs in the same interference region, the protocol begins with a period of spectrum sensing. If the channel is idle then the SU node can opportunistically access the channel by immediately assembling and transmitting a standard frame. If the channel is busy then the $\mathrm{SU}$ must determine if the PU is idle, since the channel may be busy due to another PU node, which would trigger a contention based back-off (part of the ASC phase of SCAP). If the PU is not idle then the SCAP will dictate its operation depending on if it is a co-access, co-ack or non-qualified node. A non-qualified node is relegated to only accessing during PU idle periods so it must return to spectrum sensing. However co-access nodes (and their respective co-ack nodes) begin the process of co-accessing the spectrum during PU transmissions using the CRR scheme of the SCAP.

The CRR phase is a modified version of a round robin scheme and is labeled with a dotted box in Fig. 4. The basic premise of CRR is that each co-access node within the interference range of a PU transmission will be given a coaccess transmission time slot. For each PU transmission the co-access nodes will essentially take turns co-accessing in order to maintain fair channel access 


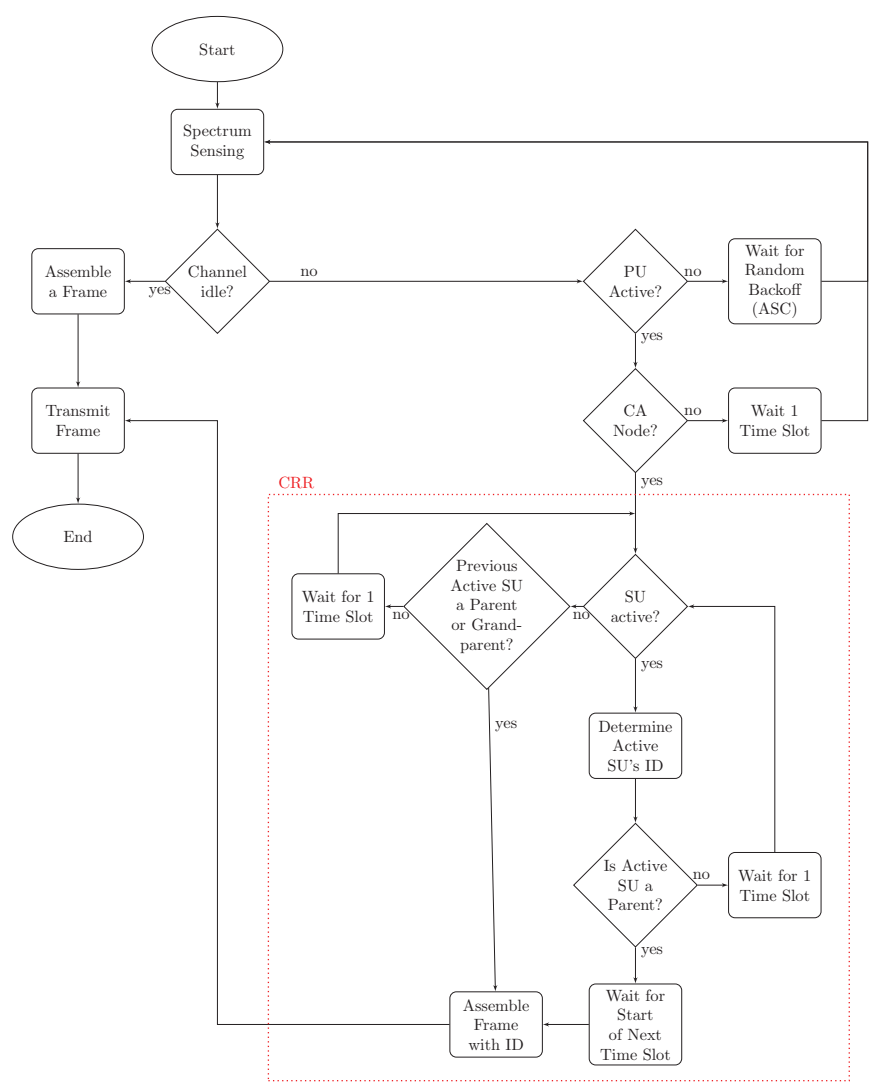

Figure 4: The operation of an SU node with the SCAP Protocol.

among SU nodes. This process of sequentially taking turns is often referred to as a round-robin scheduling scheme. The key to the CRR phase is the detection of a co-access node's turn to transmit. This is done through the use of unique identification numbers (IDs), and each node knowing the ID of the co-access node is scheduled to transmit immediately before. The details of the scheme are presented in the following subsections.

\subsection{Spectrum Detection and Initiating SCAP}

When an SU node has data to send, it begins the SCAP protocol by performing detection of the PU and any additional SU nodes. If the SU node has recently accessed this same spectrum then the detection time will be minimal. Once the SU node has determined other active SU nodes it will determine if it 
has already been a member of the SU network or if it needs to join the network.

Subsequently new SU nodes will begin estimating channel state information to categorize themselves as co-access, co-ack, or non-qualified nodes based on the parameters listed in the preceding section. We assume that the PU relay nodes are stationary. Hence it is possible within a few channel accesses to estimate the channel gains based on location and standard channel fading models. Once a node has categorized itself, then initiating channel access can begin based on the SU node characteristics. The non-qualified nodes will be relegated to participating only in the ASC phase using Carrier Sense Multiple Access With Collision Detection (CSMA/CD), and co-access/co-ack nodes will begin the round robin co-access in the CRR phase.

\subsection{Co-access Round Robin (CRR) Phase}

The scheduling of co-access nodes transmissions is done based on a round robin access scheme. In round robin scheduling each node is assigned a time slot and takes turns transmitting so that every node has equal access to the channel. The CRR scheme of the SCAP protocol is adapted from this basic scheme, but uses decentralized scheduling and prevents unused SU time slots, to result in effective and efficient spectrum co-access. In the CRR scheme, each PU packet transmission is treated as one time slot.

The logic flow of the CRR scheme is shown in Fig. 4 in the dotted box. First to facilitate the co-access nodes taking turns for co-accessing, the nodes will rely on IDs and the knowledge of which two co-access nodes have scheduled access immediately before it. The node that should co-access in the time slot immediately before a node is considered its parent and the node two time slots before it is considered its grandparent. This information is determined during the CRR group joining phase and each co-access node adds its own ID to its frame before preparing it for transmission.

During the CRR phase, the co-access node must first determine if another co-access node is currently active. If it is, then the co-access node decodes the ID from the transmission and determines if the active node is its parent. If yes, this indicates that it has the next time slot for co-access. At the beginning of the next PU co-access time slot it will assemble its data frame including its ID, 
and transmit it. If the active node is not the parent of this co-access node, then the co-access node continues to listen until it detects its parent transmission.

In the case that a co-access node scheduled for a time slot does not have data to send, the CRR scheme also implements a shifted access scheme to allow the next co-access node that is scheduled to transmit in the next time slot to access this time slot. Specifically, if the last co-access transmission time slot was for the grandparent of a co-access node, then if it detects an open co-access time slot, i.e., there is no data transmission, it accesses spectrum in this slot. Since all of the co-access nodes for a particular CRR group are within each other's interference range, the co-access node can detect absence of a predecessor node in a time slot quickly. Furthermore, this detection can be done in only a few code words. Hence, the co-access node will be able to pick up the open time slot unused by its predecessor in the round robin scheduling. For example, in Fig. 5, SU1, SU2, and SU3 take turns to co-access spectrum with the PU, but SU2 does not have data to send. SU3 detects the absent transmission by SU2, and begins spectrum co-access at the second time slot that was supposed to be used by SU2. This essentially shifts the round robin scheme to skip the turn of the absent co-access node without losing a co-access time slot.

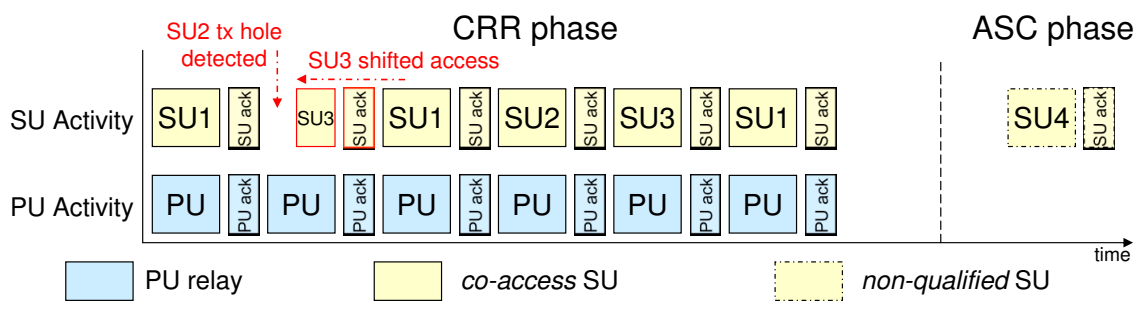

Figure 5: Co-access node (SU3) shifting in CRR after detecting an open time slot

It should be noted that the success of the CRR scheme is due to the co-access constraints that guarantee that all qualifying co-access nodes with specific PU relay will be within each other's transmission region. This means that there will be no hidden terminal possible during co-access node transmissions. This key feature makes a round-robin scheme possible because every co-access transmission can be decoded. Since every co-access node is able to decode every other qualifying co-access transmission, no extra message passing is necessary. The 


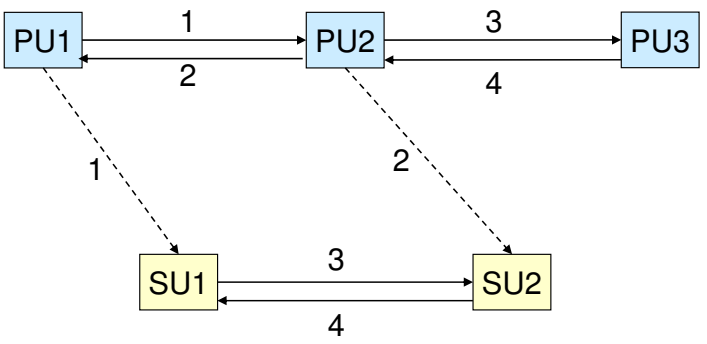

Figure 6: SU co-access transmission sequences

co-access node is transmitting this scheduling information simultaneously with the PU transmission. The PU is aware of the transmission but does not need to change its own access. Hence such spectrum co-access by SUs is considered a transparent SU control channel.

\subsubsection{Co-Access Transmission Sequence}

The co-access transmission between $\mathrm{SU}$ nodes requires a basic four step process as described in the following using the example topology in Fig. 6. First, SU1, a co-access node, will listen for PU1 transmission. Second, during the subsequent PU ACK from PU2, the co-access node's destination node (SU2 in Fig. 6) will be able to overhear the ACK. Third, when the PU relay (PU2) begins access, the first co-access node (SU1) can simultaneously co-access spectrum. Finally, in the fourth step, when the second PU node (PU3) transmits the ACK packet, the co-access or co-ack node (SU2) can simultaneously acknowledge the first co-access transmission from SU1.

Although the PU ACK packets may be somewhat different due to MAC layer addressing, they have the same basic structure. Hence SUs will be able to estimate the PU ACK packets. This may require listening for a few time slots for the correlation between the PU ACK packets in steps two and four. Since these are typically simple packets of only one or two code words, estimation is simple.

\subsubsection{Joining a CRR Group}

The CRR scheme schedules a group of co-access nodes to co-access spectrum with the PU transmissions in a round robin mode as shown in Fig. 3. A new 
co-access node requires two things to join a CRR group. First, the node will need to be assigned an ID to distinguish itself from other SU nodes. This can be initially selected as a random number or a hash function of the unique MAC address. Secondly, it must be made aware of its order in the round robin scheme. Specifically, it needs to be aware of the co-access node that is scheduled immediately before it. This process is accomplished as follows. During the first PU transmission slot where the scheduled co-access node does not have traffic, a joining co-access node will issue a beacon message which will be detected by all the current co-access members in the CRR group. The co-access node with the next scheduled transmission slot responds to the joining co-access node with an acknowledgment that its ID is unique, and with the ID of its predecessor (which becomes the predecessor of the joining co-access node). The joining coaccess node will become the current co-access node's predecessor. Note that this is analogous to adding and removing members from a linked list. Fig. 7 illustrates the process that a new co-access node, SU5, joins a CRR group. At its scheduled transmission slot, the co-access node SU3 in the current CRR group does not have traffic to send. Then the new co-access node, SU5, issues a beacon message. The co-access node scheduled to transmit in the next slot, SU4, responds to SU5 with its ID and the ID of its parent, SU3. Now, SU5 becomes the new parent of SU4, and the original parent of SU4 (i.e., SU3) becomes the parent of the joining node, SU5, and a new CRR group is formed. In case that there is no slot that a scheduled co-access node in the current CRR group does not have traffic, then the new co-access node can wait until the following ASC phase to join the CRR group.

Co-access nodes access scheme in a round robin mode by using their assigned node numbers to access the scheduled PU transmissions. This is a variation of the classic round robin scheduling algorithm which ensures max/min fairness. Thus if a co-access node has data to transmit, it must do so on the PU frame immediately following the co-access of its predecessor.

\subsubsection{Leaving a CRR Group}

A co-access node can leave a CRR group in two ways. First it can issue a termination message during its co-access slot. This message will contain its 


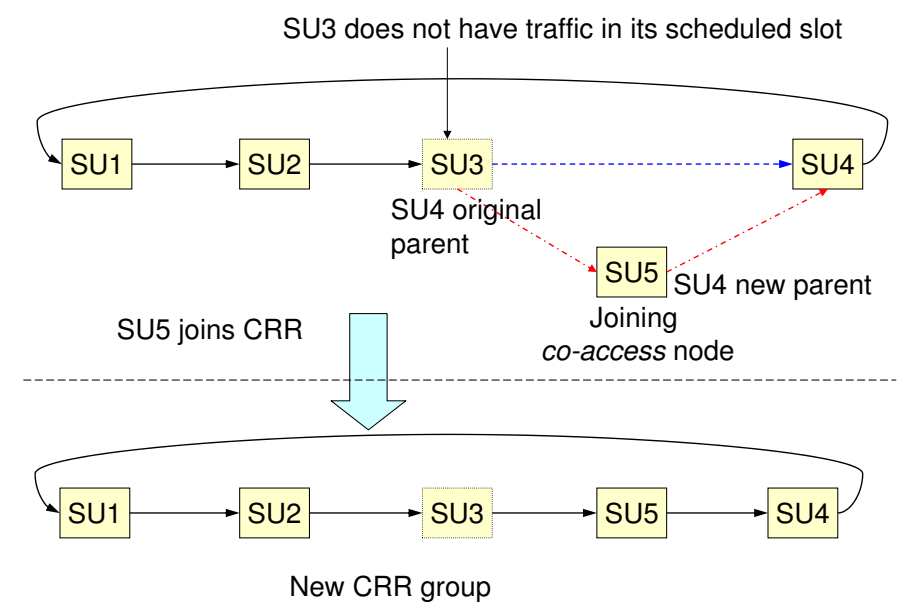

Figure 7: A co-access node joins a CRR group.

predecessor co-access node, which will be used by its successor co-access node as the new predecessor. Thus with only a single control message the CRR scheduling remains full and the old co-access node is forgotten. The other method for leaving is if a co-access node has not used its co-access slot in the CRR scheme for a given period of time, then the other co-access nodes will automatically assume this co-access node is gone and remove it from the CRR scheduling. This will require no control messages, but could result in missed transmission opportunities for other co-access nodes.

\subsection{All SU Contention (ASC) Phase}

During periods when the PU is idle, the SUs will access the licensed spectrum using an adapted contention algorithm based on CSMA with exponential backoff. Although all SU nodes will be able to access these gaps in PU transmissions, the ASC stage of the SCAP protocol focuses on providing fairness among the SU nodes. Specifically since co-access/co-ack nodes are able to transmit while the PU is active, non-qualified nodes need to be prioritized for access during the PU absence. Since CSMA with exponential backoff is a widely accepted decentralized access approach, we look to modify it to prioritize non-qualified access. A more accurate description would be to reduce the priority of SU nodes that have recently participated in co-access through increasing their default contention window. As shown in Fig. 4, when a co-access node is able to 
successfully transmit during CRR, it must automatically increment its exponential backoff for ASC stage. The more successive CRR accesses for a co-access node, the lower its priority during the next ASC stage. This window value is reset only after a successful access in the ASC phase. This results in non-qualified nodes having a higher probability of accessing the spectrum before co-access or co-ack nodes during PU absences and subsequently provides an increased fairness among all SU nodes in accessing the licensed spectrum.

\subsection{Benefits of $S C A P$}

The effect of the SCAP protocol attempting to maximize co-access opportunities has significant benefit for both the SU nodes and the PU nodes. The obvious effect for the SU nodes is the gained opportunity to transmit using licensed spectrum. However the PU effects are no less compelling. Specifically since the co-access nodes are required to meet the co-access constraint $K$ when transmitting their packets, the benefit to the PU nodes was shown as an increase in SINR. This increase in SINR will result in two possible benefits for the PU. First the PU will experience a lower number of bit errors in transmissions which will result in a lower number of corrupted packets. This in turn will reduce the number of retransmissions that are required by the PU. This reduction in retransmissions will result in either an increase in PU throughput or more likely a reduction in PU channel access. The latter is especially important since it will give more opportunities for non-qualified nodes to access the licensed spectrum during the increased availability of PU idle time slots.

Another potential benefit for PUs resulting from the increased SINR is an increased transmission range. Practical wireless communication links are given a minimum bit error rate that must be achieved in order for communications to be considered feasible. Since one of the primary factors in bit error rate is the signal degradation due to path loss, the increase in signal strength from co-accessing SUs would be able to offset an increase in path loss from moving the PU receiver farther away from the PU transmitter. Although PU wireless link engineers may be hesitant to include the benefits of SU node activity in site planning relay towers, this increased transmission range is very useful in lasthop communications. As an example with a PU digital TV transmission, the 
SU co-access would result in customers farther away being able to successfully receive the transmissions. The increased coverage area is an useful incentive the SCAP protocol and DSCA architecture can give to PU relay networks.

\section{Performance Evaluation}

We use simulations to evaluate the performance of the proposed SCAP protocol. Of interest is the specific impact the CRR component of the SCAP protocol will have on the overall performance of the SU network. We consider a 37 node PU network organized over a $600 \mathrm{~m} \times 600 \mathrm{~m}$ area in a cellular grid topology. The PU traffic is broadcast from the central tower to the entire network through multihop relay. Each PU and SU node operates with an omnidirectional antennae and a broadcast power of 0.5 Watt with a channel bandwidth of $20 \mathrm{MHz}$ based on the WiFi model. The PU nodes are assumed to be stationary and follow a channel access scheme of broadcasting 500-byte frames with channel slot duration of 1 milliseconds (ms). SU nodes are uniformly and randomly deployed across the network. We assume the packet generation at each SU node follows a Poisson process with the mean inter-packet arrival time as $50 \mathrm{~ms}$. The $\mathrm{SU}$ frame is also assumed 500 bytes. Furthermore, we assume that in all experiments, 10 percent of PU nodes are active if not otherwise noted. Finally, we consider the required PU co-access constraint. Specifically, we require $K=0.1$ or $10 \%$ increase of SINR at the PU receiver. The SUs require $90 \%$ power to be used for $\mathrm{SU}$ packet transmission, i.e., $\gamma \geq 0.9$. A path loss of $d^{-3}$ is assumed, where $d$ is the distance.

First we look at how well the proposed SCAP protocol supports a large number of SUs. As shown in Fig. 8, we simulate the average throughput an SU node acquires using the SCAP protocol. For comparison, we consider an opportunistic spectrum access (OSA) protocol which assumes that the SU nodes can instantly and perfectly detect PU access and absence. From Fig. 8 we find that the SCAP protocol clearly outperforms the OSA protocol, with a large margin on throughput. Furthermore, when the number of SUs increases, the performance gap is even larger. This is because with more SU nodes, the contention for spectrum access to the channel idle periods is more severe under 


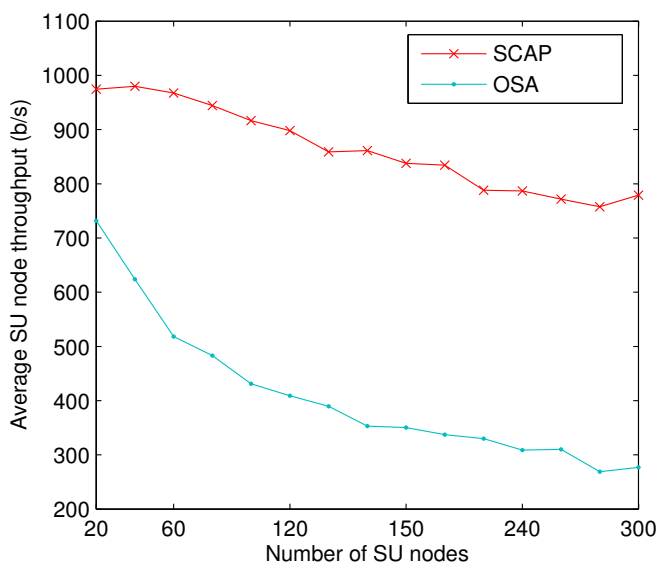

Figure 8: Average SU node throughput

the OSA protocol. In contrast, the SU nodes can co-access spectrum together with PU nodes under the SCAP protocol; hence the contention for the channel idle periods does not increase as fast as in the case of the OSA protocol.

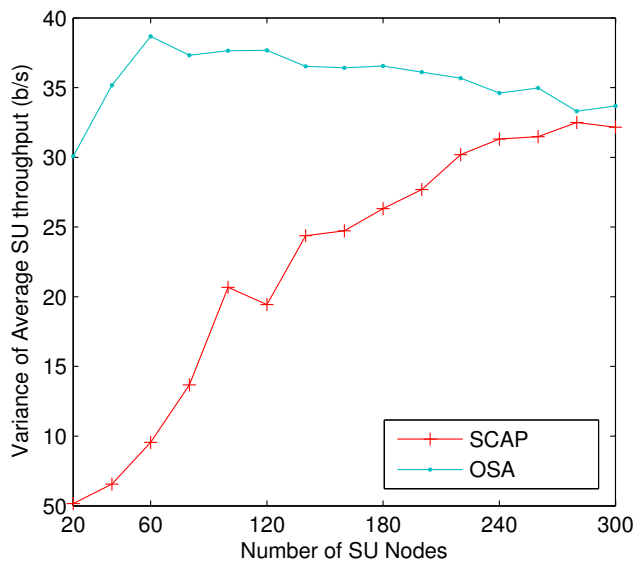

Figure 9: Variation of SU node throughput

Fig. 9 illustrates the variation of SU per-node throughput, i.e., the difference between the maximum and the minimum throughput among the SU nodes. SCAP has a smaller variation for the SU node throughput, especially when the number of SU nodes is small. This means SCAP achieves better fairness 


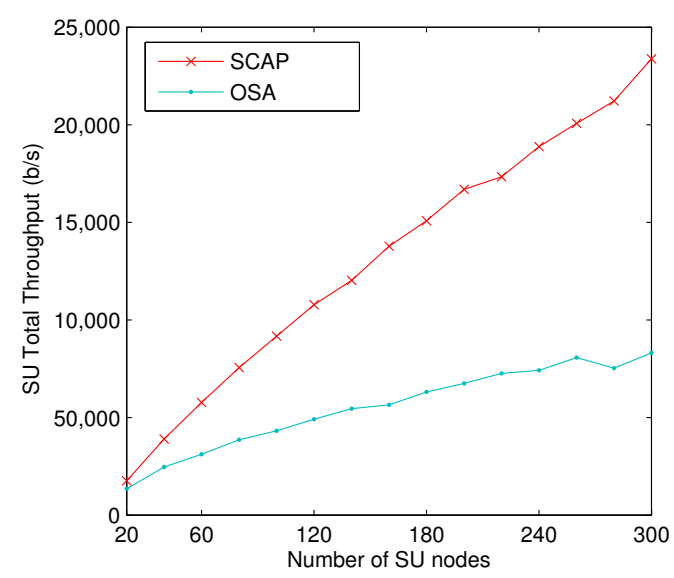

Figure 10: Total SU node throughput

on throughput for the SU nodes. In Fig. 10 we present the performance of the entire SU network. Of considerable note is that the SCAP protocol has a higher system throughput than the OSA protocol. This is due to the increased spectrum efficiency of the CRR component in the SCAP protocol. Specifically, the SCAP protocol utilizes the spectrum access during the PU activity period much more efficiently.

Fig. 11 illustrates the mean channel access delay for the SU nodes, as a function of the PU activity, where " $z \% \mathrm{PU}$ active" indicates that $z \% \mathrm{PU}$ nodes are active at any time. This figure clearly indicates the benefit of the SCAP protocol. While there are more active PU nodes, by intuition, the spectrum access opportunity for SU nodes decreases; as a result, the channel access delay increases. This is exactly what happens to the OSA protocol. However, for the SCAP protocol, the channel access delay does not increase, but decreases. This anti-intuition observation is due to the spectrum co-access feature of the SCAP protocol. This is because more active PU nodes means more opportunity for spectrum co-access by SUs. In other words, more SU nodes become qualified for spectrum co-access with PU nodes, since with more active PU nodes, it is clear that more SU nodes are close to the active PU nodes and can meet the constraints for spectrum co-access. Thus, the mean channel access delay decreases since more SU nodes can access the channel immediately when they 


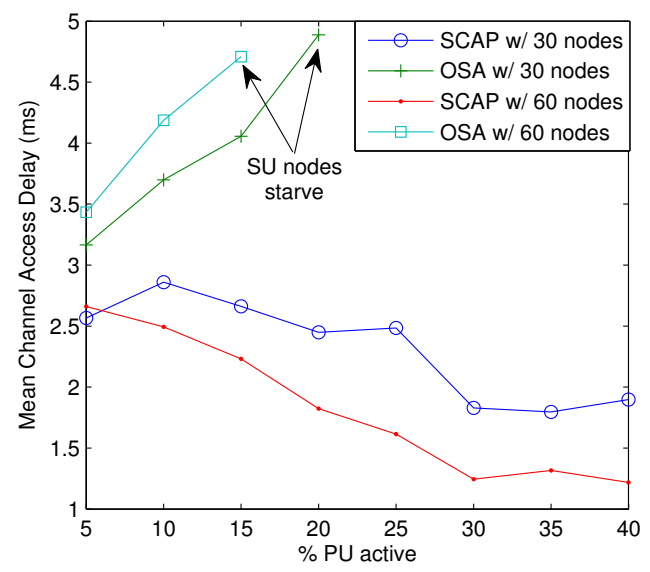

Figure 11: Mean channel access delay as PU activity increases

have traffic to transmit.

One key benefit of the presence of SU co-access nodes is the potential reduction in packet error rate of the PU links. In Fig. 12 we compare the co-access effect on the PU links with respect to commonly employed modulation schemes to gain an understanding of how much the $\mathrm{SU}$ co-access could reduce the number of dropped packets in the PU link. Since many PU links will have potentially high data rates, even small decreases in packet error rate can result in important PU network performance increases. For example with the 64 PAM modulation and the $100 \mathrm{~m}$ distance between PU nodes, a co-access link with the PU co-access constraint $K=0.2$ decreases the packet error rate from $0.25 \%$ to $0.041 \%$. The results can vary with modulation schemes, but improvements are consistently shown.

Since the transmit power of both the PU and the SU links are typically regulated, another important interpretation of the $\mathrm{SU}$ co-access is that this can be used as a tool for increasing the possible range of PU nodes. In Fig. 13 we consider this range increase by looking at the effect of increasing the PU coaccess constraint $K$ from 0 to 1 . In this figure we have labeled the standard bit error rate minimum limit at $10^{-5}$. This is the minimum threshold that many digital communication links must meet when they are designed for wireless communications. Therefore it gives a good understanding of the maximum 


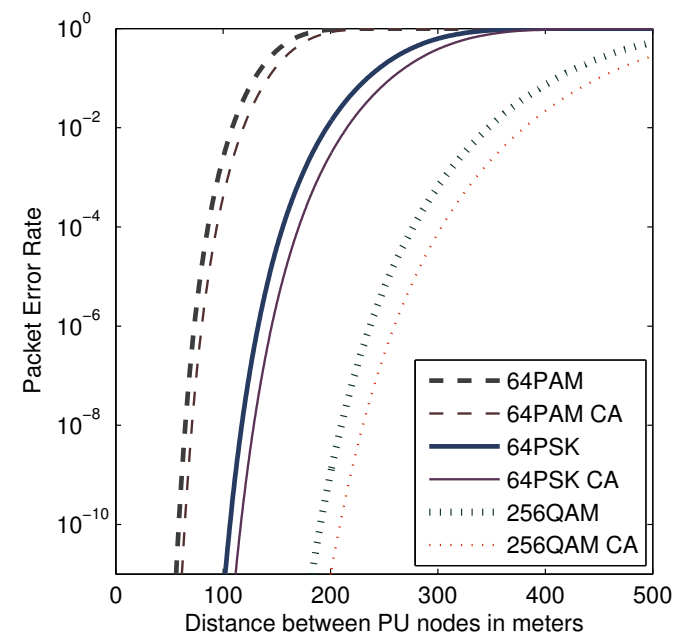

Figure 12: PU packet error rate without or with co-access (CA) under different modulation schemes

distance of PU links that can be supported. For example, with the 256 symbol QAM modulation scheme, the maximum distance for a PU link without SU co-access is 297 meters. However with co-access constraint $K=0.4$, the limit is increased to 351 meters. Therefore PU networks could leverage SU co-access into either reducing packet error rates or increasing coverage area.

\section{Conclusion}

In this paper, we have proposed a spectrum co-access protocol (SCAP) for the DSCA architecture in our earlier work. The SCAP protocol tries to find spectrum co-access opportunities for qualified secondary user (SU) nodes while give spectrum access opportunities to the SU nodes that are not qualified for spectrum co-access with PU nodes. With the SCAP protocol, if there are active PU transmissions, SU nodes co-access spectrum using a round robin scheme termed Co-access Round Robin (CRR), while if the PUs are idle, SU nodes compete for spectrum access fairly with a scheme termed All SU Contention (ASC). The performance evaluation indicates that the SCAP protocol significantly outperforms the opportunistic spectrum access protocol that was widely assumed for cognitive radio networks. 


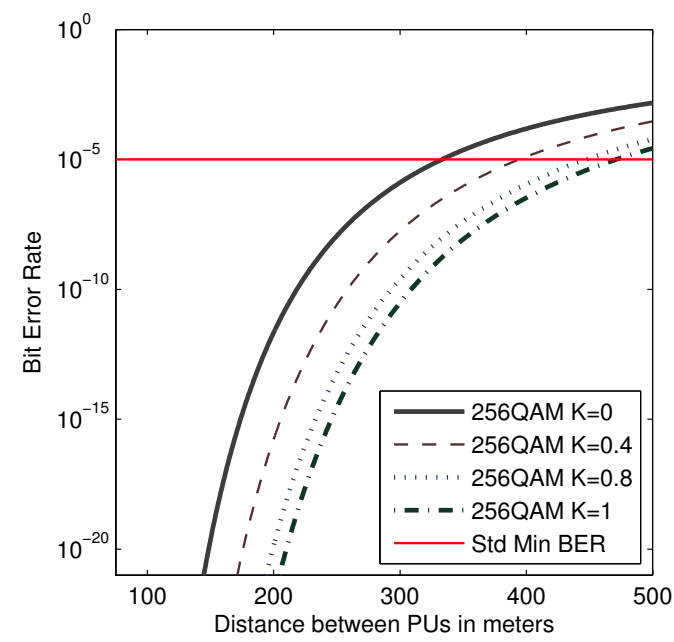

Figure 13: Bit error rate for PUs with different co-access constraints $(K)$

\section{Acknowledgments}

The research of C. Xin is supported in part by NSF under grants CNS1418012 and ECCS-1418013. The research of M. Song is supported in part by NSF Career Award CNS-1248092.

\section{References}

[1] J. Backens, M. Song, and C. Xin, "A transparent spectrum co-access protocol for cognitive radio networks," in Proc. International Conference on Computer Communications and Networks (ICCCN), 2014.

[2] X. Feng, Q. Zhang, and J. Zhang, "Hybrid pricing for TV white space database," in Proc. IEEE Infocom, 2013.

[3] M. Caleffi and A. S. Cacciapuoti, "Database access strategy for TV white space cognitive radio networks," in IEEE International Conference on Sensing, Communication, and Networking Workshops (SECON Workshops), 2014. 
[4] A. Mancuso and B. Patil. (2013, May) Protocol to access white-space (PAWS) databases: Use cases and requirements. IETF RFC 6953. [Online]. Available: http://www.ietf.org

[5] P. Bahl, R. Chandra, T. Moscibroda, R. Murty, and M. Welsh, "White space networking with wi-fi like connectivity," in Proc. ACM SIGCOMM, 2009, pp. 27-38.

[6] Y. Yuan, P. Bahl, R. Chandra, T. Moscibroda, and Y. Wu, "Allocating dynamic time-spectrum blocks in cognitive radio networks," in Proc. ACM MobiHoc, 2007, pp. 130-139.

[7] Y. Yuan, P. Bahl, R. Chandra, P. Chou, J. Ferrell, T. Moscibroda, S. Narlanka, and Y. Wu, "KNOWS: Cognitive radio networks over white spaces," in Proc. IEEE DySPAN, 2007, pp. 416-427.

[8] Q. Yan, M. Li, F. Chen, T. Jiang, W. Lou, T. Hou, and C.-T. Lu, "Nonparametric passive traffic monitoring in cognitive radio networks," in Proc. IEEE Infocom, 2013.

[9] Y. Zhang, G. Yu, Q. Li, H. Wang, X. Zhu, and B. Wang, "Channel-hoppingbased communication rendezvous in cognitive radio networks," IEEE/ACM Transactions on Networking, vol. 22, no. 3, pp. 889-902, Jun. 2014.

[10] W. Li, X. Cheng, T. Jing, and X. Xing, "Cooperative multi-hop relaying via network formation games in cognitive radio networks," in Proc. IEEE Infocom, 2013.

[11] Q. Yan, M. Li, T. Jiang, W. Lou, and Y. Hou, "Vulnerability and protection for distributed consensus-based spectrum sensing in cognitive radio networks," in Proc. IEEE Infocom, 2012.

[12] Y. Liu, L. Cai, and X. Shen, "Spectrum-aware opportunistic routing in multi-hop cognitive radio networks," IEEE Journal on Selected Areas in Communications, vol. 30, no. 10, pp. 1958-1968, Nov. 2012. 
[13] M. Pan, P. Li, Y. Song, Y. Fang, and P. Lin, "Spectrum clouds: A session based spectrum trading system for multi-hop cognitive radio networks," in Proc. IEEE Infocom, 2012.

[14] C. Tekin and M. Liu, "Approximately optimal adaptive learning in opportunistic spectrum access," in Proc. IEEE Infocom, 2012.

[15] A. De Domenico, E. C. Strinati, and M. Di Benedetto, "A survey on mac strategies for cognitive radio networks," IEEE Communications Surveys $\&$ Tutorials, vol. 14, no. 1, pp. 21-44, 2012.

[16] J. Jia, Q. Zhang, and X. Shen, "HC-MAC: A hardware-constrained cognitive MAC for efficient spectrum management," IEEE Journal on Selected Areas in Communications, vol. 26, no. 1, pp. 106-117, Jan. 2008.

[17] B. Hamdaoui and K. Shin, "OS-MAC: An efficient MAC protocol for spectrum-agile wireless networks," IEEE Transactions on Mobile Computing, vol. 7, no. 8, pp. 915-930, Aug. 2008.

[18] H. Su and X. Zhang, "Cross-layer based opportunistic MAC protocols for qos provisionings over cognitive radio wireless networks," IEEE Journal on Selected Areas in Communications, vol. 26, no. 1, pp. 118-129, Jan. 2008.

[19] Z. Lin, H. Liu, X. Chu, and Y.-W. Leung, "Jump-stay based channelhopping algorithm with guaranteed rendezvous for cognitive radio networks," in Proc. IEEE Infocom, 2011, pp. 2444-2452.

[20] K. Bian, J.-M. Park, and R. Chen, "A quorum-based framework for establishing control channels in dynamic spectrum access networks," in Proc. ACM MobiCom, 2009, pp. 25-36.

[21] N. Theis, R. Thomas, and L. DaSilva, "Rendezvous for cognitive radios," IEEE Transactions on Mobile Computing, vol. 10, no. 2, pp. 216-227, Feb. 2011.

[22] J. Backens, C. Xin, M. Song, and C. Chen, "DSCA: Dynamic spectrum coaccess between the primary users and the secondary users," IEEE Transactions on Vehicular Technology, vol. 64, no. 2, pp. 668-676, Feb 2015. 
[23] M. Costa, "Writing on dirty paper (corresp.)," IEEE Transactions on Information Theory, vol. 29, no. 3, pp. 439 - 441, may 1983. 